\title{
Genetic services in Britain: a strategy for success after the National Health Service and Community Care Act 1990
}

\author{
Rodney Harris
}

The reform of the British National Health Service will bring radical changes in the way existing services and new developments are funded, although it is intended that funding will still be through general taxation. Health authorities will themselves assess the health needs of their resident populations and will then negotiate contracts to purchase the services which they expect will achieve the optimum improvement in health. The emphasis is on quality of care and value for money with new audit and resource management systems to monitor quality, keep accounts, and reimburse service providers.

Because the reforms emphasise preventive medicine, they should favour the development of clinical and laboratory genetics. Indeed the changes offer the opportunity to work constructively in the development of effective plans with general managers, directors of public health, and family practitioners. Purchasers will require criteria for assessing genetic needs and geneticists will have to audit the quality, quantity, and cost of their work.

This paper discusses how health authorities might assess the genetic needs of their resident populations and how geneticists might show the quality and quantity of the services that they provide. The difference between the population needs identified by health authorities and the current provision will constitute the basis for the planning of service developments.

\section{Medical genetics and the National Health Service (NHS)}

Medical genetics is a rapidly developing specialty which has successfully introduced clinical, cyto-

Department of Medical Genetics, St Mary's Hospital, Hathersage Road, Manchester M13 0JH.

R Harris genetic, and molecular diagnosis within the context of fully informed, non-directive counselling. These bring relief from anxiety to many individual people and families, complemented by accurate prenatal and postnatal diagnosis and carrier detection for a rapidly increasing range of genetic diseases. Population screening and prenatal diagnosis for haemoglobin disorders and Tay-Sachs disease are successful in specific groups, while screening for carriers of cystic fibrosis, which is currently being evaluated, may involve the entire British population of reproductive age. Many common diseases of adult life have an important genetic component in their aetiology; molecular genetics thus has implications for many specialties and for preventive medicine.

Genetic procedures have been extensively evaluated. The Royal College of Physicians of London reported on prenatal diagnosis and genetic screening. ${ }^{1}$ The Department of Health commissioned a continuing independent evaluation of DNA services in three centres (Manchester, Cardiff, and the Institute of Child Health, London) which has reported favourably. ${ }^{2}$ The Department of Health also signalled its approval after the medical royal colleges 'Joint Statement' in 1987, which recommended integrated regional genetic centres combining clinical and laboratory genetics "under one roof".

There have been further discussions about NHS genetics since the White Paper Working for patients was published in 1988. These have involved the medical royal colleges, the Department of Health, and the professional societies. In her constructive reply to the Clinical Genetics Society, the health minister gave a particularly important guide to Departmental thinking. It is now understood that regional directors of public health will discuss collectively the question of a national network of regional genetic services and the need for a rational plan for the delivery of molecular genetics diagnostic services. 
THE PRESENT SITUATION

\section{Manpower}

Because of quotas of clinical training grades and changes in the NHS, the Royal College of Physicians of London (Report on Clinical Genetics in the 1990s and Beyond. Royal College of Physicians of London Committee on Clinical Genetics; in preparation) surveyed clinical manpower in genetic centres while the Clinical Genetics Society asked its members in each region about the current state of planning.

Trained manpower numbers are very limited: there are the equivalent of only 157 whole time doctors and clinical coworkers in clinical genetic centres in the UK, excluding laboratory scientists. This is the equivalent of one worker per 18000 genetic patients assuming the lowest prevalence figure of $5 \%$ for genetic disorders quoted by Baird et al. ${ }^{3}$

\section{Regional organisation of genetic services}

Most regional health authorities appear to recognise the merits of integrated clinical and laboratory services. For example, one regional genetic service is to be retained even though more than 100 services in other specialties are to lose their regional status. Genetics is the only service identified by that region as a "... new specialty with considerable potential for rapid expansion and major implications for a wider range of other services ...". Other centres have informal agreements with regions that the clinical and laboratory components should not be taken apart because of the loss of close cooperation and coordination that would result.

Yet even before the NHS and Community Care Bill became law, an effect of imminent change has been to halt new developments. There is much uncertainty about the nature of proposed contracts with health authorities and continuing concern about underfunding. Although money will come according to the ability of providers to attract it, if medical genetics enters the new environment with markedly inadequate resources there will be little prospect of offering the sort of service which would attract expansion in a competitive market. Most regional health authorities are already financially overstretched and many geneticists believe that there will have to be curtailment of services if development monies cannot be found.

It is encouraging that managers have already shown their willingness to try to rectify anomalies. These have included the halting of a proposed 'de-designation' of a regional genetic service and the correction of a failure to advertise a regionally funded consultant clinical geneticist post which was subsequently filled. In another region the director of public health is actively intervening to prevent the reduction in service that would follow the abandonment of long established recharging arrangements between districts.
Incipient privatisation of a genetic service is described in one region in relation to serum testing for Down's syndrome, where a district health authority is reported to be paying a private laboratory with NHS $\stackrel{\vec{\rho}}{\rightarrow}$ funds for NHS patients to provide both serum tests and the culture of amniotic fluids. Although this is currently giving cause for concern, after April 1991 the law will require that the health authority monitor the quality of the service. The local director of public health will ensure that a 'screening' service will not be provided on request rather than on a population basis and the contract will specify that this new procedure will be subjected to systematic follow up and evaluation of its consequences with adequate specialist counselling and backup services for those screened.

\section{Towards contracting for genetic service agreements}

For the first year of contracting, 1991/2, most regions will probably act as broker between the provider genetic units and the purchasing health districts. However, the general intention is to move rapidly towards a contracting system in which district health authorities will decide how much to spend on genetic services to meet the needs of their resident populations. Some managers, anticipating the need, have requested that the number of patients seen each month and their post codes be recorded, allowin districts of referral to be identified.

Although there are two parties to a formal contract 1991/2, the first year of operation, will not be typical and providers may not have as much involvement in negotiations as in subsequent years. For example, in one region a draft contract is said to be "... very sensible in principle, but is uncosted and not very detailed . . . the real 'catch 22 ' is that the contract is based on the assumption that last year's resources were adequate for last year's workload when in fact resources were $25 \%$ deficient".

One genetic centre plans to offer a comprehensive genetic service with a single clinical and laboratory regional budget based on a per capita calculation. Another region has agreed informally that 'community genetics' is a preventive exercise for which funding should be top sliced. Nevertheless, at this stage few if any regional health authorities have confirmed these approaches and it is likely that regions will not be able to top slice monies without district health authority approval. Even for 1991/2, districts in one region are refusing to agree top slicing to fund developments in regional services because of their current and likely future overspending.

The high turnover of new genetic patients might be expected to be financially advantageous for selfgoverning trusts, who should therefore be singing their praises. However, in one centre application documents to form a self-governing trust failed even to mention the clinical genetic services available. The 
senior clinical geneticist involved believes that this may be because the service is very inexpensive to run and undemanding of nursing resources and expresses apprehension about the level of understanding of genetics at management level.

\section{Genetic services: a strategy for success}

Several important aspects that have been emphasised in the health service changes, including preventive medicine, clinical audit, quality of care, and value for money, are strongly supported by geneticists. However, most centres do not have appropriate data for contracting and there is a general poverty of hardware and software for general audit and resource management. Geneticists are not alone in being conscious of a lack of experience in planning business strategies and there is unease at the amount of professional time which will be diverted from clinical work. Nevertheless, the preparation of service (and development) plans must be professionally guided because funding for health services will in future depend on health authorities' interpretations of the correct balance between population need and the quality and quantity of the service being provided. The decisions that health authorities make will be greatly influenced by the resources available, value for money (cost-effectiveness), and evidence of beneficial outcomes. Genetic services will be better able to flourish in this new environment if the responsibilities of health authorities are clearly understood; they have a need for coherent and persuasive service plans, supported by appropriate data, with which to negotiate contracts.

\section{Responsibilities of the new health authorities}

The prime responsibility of the new district health authorities will be to purchase those services which achieve the optimum improvement in the health of their populations by: (1) assessing the health needs of their resident populations, (2) reviewing the service options for meeting those needs, (3) specifying the preferred pattern of service provision, (4) placing contracts for services, (5) monitoring the implementation of those contracts, (6) monitoring the health of the population, and (7) controlling expenditure within cash limits.

Plans describing well organised genetic services will provide district health authorities with a clear service option. Where regions act as brokers a possible pattern of provision will consist of a single service agreement, between region and the regional genetic service, but only after region has consulted districts. Monitoring the implementation of contracts and the effects upon health of the population are key activities which will require much discussion on the criteria to be adopted and the data to be provided by genetic centres (see providers' guidelines below). Identified resources will not yet have been set by districts for genetic services because health authorities have not yet assessed the genetic health needs of their populations. Accordingly these may not be given a high priority and without appropriate information health authorities may underestimate the need.

\section{Purchasers' guidelines}

Purchasers' guidelines will help district health authorities when they are assessing the genetic needs of their resident populations based on: (1) the population prevalence of relevant disorders, (2) the screening, diagnostic, and support measures available, and (3) outcomes which achieve the optimum improvement in health. Individual health authorities could request this information to use as a yardstick when they assess for themselves what genetic services they require to meet the needs of their populations taking into account demographic factors such as fertility, maternal age, and ethnicity.

\section{Providers' guidelines}

Because there are too few trained geneticists for the workloads generated by the new technology, and by greater public awareness, an increasing proportion of clinical genetic work will be done by non-geneticists. Whether district health authorities purchase genetic services from regional genetic centres will require unambiguous definitions of the several specialist roles that contribute to effective genetic services and the quality and cost of the service offered.

The unique features of genetic services will generate special needs in resource management. Medical genetics is rapidly developing, is regionally organised, and combines specialised, integrated clinical and laboratory components. Genetics is also unusual in dealing with families and not just with individual patients; it is concerned with reproductive decisions requiring informed choices by clients; it requires care to protect confidentiality and a particularly sensitive awareness of ethical issues. This complexity usually requires records to be held within genetics departments. Genetic records are nonstandard and contain information on many relatives over several generations. These specialised records are rarely serviced by hospital records departments and genetics data are not included in Korner returns. Clinical genetic workloads are thus grossly underreported. No standardised computer system has been agreed for clinical data storage (work is in hand to make good this deficiency-see below).

It is not surprising that few genetic providers of services could immediately document evidence of beneficial outcome, workload, and costs for each of the districts they serve and would have difficulty in 
negotiating service agreements on the basis of current activities. Since neither providers' nor purchasers' guidelines exist, most medical genetic services could at the moment be contracted neither on the basis of purchasers' need nor on systematic district related current workloads.

In fact the 1991/2 arrangements for genetics services are likely to be based on the 1990/1 budget, although this will be called 'contracts'. There is not enough time before April 1991 for more sophisticated service agreements because authorities are required to achieve a 'seamless' transition to contracts with no loss of services. However, negotiations for service agreements will soon begin for which both purchasers' and providers' guidelines will be needed. A joint approach by all clinical genetic and laboratory services in the region will be important to preserve the integrated nature of the service. Health authorities will also find it easier to agree block contracts for genetic services rather than to try to sort out allocations to individual clinical and laboratory services, particularly where the regional service is integrated and each component of the service supports the other.

\section{Future developments will depend on purchasers' and providers' guidelines}

Comparison of purchasers' and providers' guidelines will inevitably show that the actual service funded is considerably less than expected from the assessed genetic needs of the population. Of course, funds are unlikely to be found immediately to satisfy these needs but the discrepancy between need and provision will be an important factor in negotiating contracts. Genetic centres that can document both population need and provision will be in an excellent position to negotiate with well informed directors of public health and will probably both maintain their current services and achieve new developments, providing these can be evaluated.

\section{Preparation of guidelines}

The Clinical Genetics Society established in 1989 a working party to advise generally on medical audit in genetics. As the present paper shows there is now an urgent need to address the specific issues of purchasers' and providers' guidelines for genetic services. The Royal College of Physicians of London Committee on Clinical Genetics has therefore convened two new working parties to advise on:

(1) Providers' guidelines to report by the end of 1990 with the following terms of reference.

To advise on a strategy for computer systems for genetic services and, by liaising with the CGS working party on audit, identify a simple, standardised data set for clinical workloads, district of origin, and costs per item and for audit.

(2) Purchasers' guidelines with the following terms of reference.

(a) Toprovide information for district health authorities to use as a yardstick when they decide for themselves what genetic services they require to meet the needs of their populations taking into account (i) the prevalence of genetic and congenital disorder, (ii) demographic factors, such as fertility, maternal age and ethnicity, and (iii) the screening, diagnostic, and support measures available to achieve the optimum improvement in health.

(b) To review reports of beneficial outcome of genetic procedures which will be of value to health authorities when they are identifying priorities.

The reports of the working parties should b\& helpful in predicting, auditing, and costing clinic $\mathbb{\Psi}$ genetic referral patterns and workloads and therefor 5 . in developing service plans. The epidemiologicaI content of the purchasers' guidelines may also help to anticipate some of the workloads for genetic laboratories and assist joint planning by professional groups directly involved in genetic services.

I am grateful to colleagues in the Health Service and the Royal College for invaluable information and advice.

1 Royal College of Physicians of London. Report on Prenatal diagnosis and genetic screening. London: RCP, 1989.

2 Rona RJ, Swan AV, Beech R, et al. Demand for DNA probes in three genetic centres in Britain (August 1986 to July 1987). f Med Genet 1989;26:226-36.

3 Baird PA, Anderson TW, Newcombe HB, Lowry RB. Genetic disorders in children and young adults: a population study. $\mathrm{Am}$ f Hum Genet 1988;42:677-93. 